\title{
Pulmonary Tuberculosis Disseminating and Presenting as Bilateral Hydronephrosis and Renal Abscess: a Potential Threat in the Era of Multi-Drug Resistant Tuberculosis MDR-TB
}

\author{
K V Ramana* \\ Department of Microbiology, Prathima Institute of Medical Sciences, Karimnagar, Andhrapradesh, India \\ *Corresponding author: ramana_20021@rediffmail.com
}

Received June 16, 2014; Revised June 26, 2014; Accepted July 01, 2014

\begin{abstract}
Mycobacterium tuberculosis, the causative agent of tuberculosis was discovered by Robert Koch in 1882. It has been observed that $30 \%$ of the world population harbours Mycobacterium tuberculosis. Spread of Human immunodeficiency virus (HIV) infection, malignancies, globalization and malnutrition are some of the major contributing factors for increase in the global burden of tuberculosis (pulmonary and extra-pulmonary TB). Dissemination/spread of tubercle bacilli from the lungs to other organs of the body either through blood or lymphatic circulation results in extra-pulmonary tuberculosis. Immunodeficiency, either due to HIV infection or malignancies, malnutrition and inadequate treatment for pulmonary TB may be considered as some of the predisposing factors for disseminated/miliary TB. It has also been noted that most cases of extra-pulmonary TB are diagnosed accidentally and after a long period of time (months to years) since recovering from pulmonary TB. In the era of Multi-drug resistant tuberculosis (MDR-TB), extremely drug resistant tuberculosis (XDR-TB) and increase in the incidences of disseminated/extra-pulmonary tuberculosis poses a challenge to the medical personnel. Among the extra-pulmonary cases of tuberculosis genitourinary tuberculosis (GUTB) appears to be most frequent as evidenced by the available literature.
\end{abstract}

Keywords: Mycobacterium tuberculosis, extra-pulmonary tuberculosis, genitourinary tuberculosis (GUTB)

Cite This Article: K V Ramana, "Pulmonary Tuberculosis Disseminating and Presenting as Bilateral Hydronephrosis and Renal Abscess: a Potential Threat in the Era of Multi-Drug Resistant Tuberculosis MDRTB.” American Journal of Infectious Diseases and Microbiology, vol. 2, no. 3 (2014): 48-50. doi: 10.12691/ajidm-2-3-2.

\section{Historical Background}

Ancient descriptions of tuberculosis (TB) as a disease named as Yaksma and Balasa in Rigveda and Atharva veda respectively, confirm the historical significance of tuberculosis. Sushrutha Samhitha, written in 600 BC notes that tuberculosis can be treated with breast milk, meat, alcohol and rest. Yajurveda, another ancient literature suggests that TB infected patients must move to higher altitudes for a possible cure. TB has also been noted as a social stigma, where in, infected population are labelled as impure and unfit for marriages as described in Manu smrithi, a scripture written around 1500 BC. Mycobacterium tuberculosis, the causative agent of tuberculosis was discovered by Robert Koch in 1882. Later in 1920, the BCG (Bacillus- Calmettee- Guierin) vaccine was available for immunization/vaccination against TB. The first anti-tubercular drug, Streptomycin was discovered in 1944. In the year 1970, the USA reported the first outbreak of Multi-drug resistant tuberculosis (MDR-TB). Reports of Extremely drug resistant tuberculosis (XDR-TB) have been noted from South African-KwaZulu-Natal province in 2005. World Health Organization (WHO) in 1993 has declared the TB as a global emergency. TB has been noted as a disease of young and middle-aged adults. Pulmonary tuberculosis, the most common clinical presentation of tuberculosis in human has no gender predilection but a 5:3 infection rate among male and female is observed.

\section{Introduction}

Tuberculosis (TB) continues to be a major public health problem prevalent throughout the world with increasing incidences in the developing countries. The WHO report on Global Tuberculosis(2013) reported that about 8.6 million new TB cases occurred in 2012 world-wide with India being the highest TB burdened country in the world, accounting for a quarter (26\%) of all TB cases [1]. It was in $1700 \mathrm{AD}$ that John Jacob Magnet identified a possible case of disseminated tuberculosis where numerous millet seed shaped lesions were observed on autopsy and coined the term military tuberculosis (miliarius in Latin meaning 
millet seed like) for dissemination of TB (http://en.wikipedia.org/wiki/Miliary_tuberculosis).

According to CDC (Centres for Disease Control and prevention) report, genitourinary tuberculosis comprises $12 \%$ to $18 \%$ of extra pulmonary tuberculosis and is considered as the most common manifestation of extra pulmonary $\mathrm{TB}$, accounting for $15 \%$ to $20 \%$ of infections [2]. It has been observed that $30 \%$ of the world population harbours Mycobacterium tuberculosis [3]. Spread of Human immunodeficiency virus (HIV) infection, malignancies, globalization and malnutrition are some of the major contributing factors for increase in the global burden of tuberculosis (pulmonary and extra-pulmonary TB) $[4,5]$. Previous reports have also noted that $2-20 \%$ of the pulmonary tuberculosis patients may develop genitorurinary tuberculosis [5,6,7]. A recent Indian study has observed that in males infected with pulmonary tuberculosis, $36 \%$ have developed genitourinary tuberculosis (GUTB) [8]. Existing literature also emphasizes that the severity of the GUTB depends on the time of diagnosis of extra-pulmonary tuberculosis which may vary from 2-15 years [9,10]. Diagnosis of disseminated TB is complex as the patients present mostly with non-specific symptoms and present late in the disease which may result in increased rates of morbidity and mortality. A combination of microscopic, cultural, radiological, endoscopic, serological and molecular methods is required for definitive diagnosis of extrapulmonary tuberculosis. This is a case of pulmonary tuberculosis disseminating and presenting as bilateral hydronephrosis and renal abscess in a young male patient.

\section{Case Presentation}

A 25-year-old adult male was admitted to hospital with complaints of continuous low grade fever since 2 months with chills, rigors and sweating. Patient complained of cough since 15 days and vomiting. He complained of persistent low back pain and occasional hematuria. Patient gave no history of shortness of breath, chest pain and haemoptysis. No history of hypertension, diabetes mellitus and coronary artery disease was present. Patient complained of loss of appetite and weight loss since 2 months. Patient was a non smoker and was diagnosed as suffering from pulmonary tuberculosis eight years back and had been on anti-tubercular drugs for a period of six months (thrice a week regimen: Isoniazid (300 mg), Rifampicin (450 mg), Pyrazinamide (1500 mg), Ethambutol (1200 mg), Streptomycin (750 mg) ) with no adequate follow up. On admission patients complete blood analysis and biochemical profile (liver function test and kidney function test) was performed. Patient was suffering from anaemia $(\mathrm{HB} \%=6.2)$, reduced red blood cell counts which were microcytic and hypo chromic $\left(2.9\right.$ cells $\left./ \mathrm{mm}^{3}\right)$, mild leucocytosis (TLC $=12900$ cells $/ \mathrm{mm}^{3}$ ) and erythrocyte sedimentation rate of $110 \mathrm{~mm}$. Liver function tests revealed increased total serum bilirubin $(2.1 \mathrm{gm} / \mathrm{dL})$ and mildly raised serum alkaline phosphatase activities (170 IU/L). Serum creatinine activity was raised to 2 $\mathrm{gm} / \mathrm{dL}$. After an informed consent and appropriate counselling, patient was tested for Human Immunodeficiency Virus (HIV), Hepatitis B and Hepatitis $\mathrm{C}$ viruses and was found to be non-reactive. An ultrasound
(USG) examination of abdomen revealed grade 1 renal parenchyma changes and bilateral hydronephrosis. USG also showed perinephric lymph nodes, debris in the pelvis of left kidney and signs of abscess in left kidney. Multiple renal and seminal vesicle cysts were also noted Figure 1. Chest $\mathrm{X}$ ray revealed pulmonary infiltrations suggestive of tuberculosis. A provisional diagnosis of bilateral hydronephrosis with renal abscess was made and pus was aspirated from the abscess site and was sent to clinical laboratory for microbiological examination. On examination the aspitaed pus was thick in texture, slightly blood tinged. Grams stain of the pus showed no bacteria and routine culture revealed no growth of aerobic/microaerophilic bacteria. Ziehl-Neelsen's staining for acid fast bacilli revealed the presence of acid fast bacteria. The routine urine culture was sterile and negative for acid fast bacilli. Sputum was negative for acid fast bacilli. A diagnosis of renal tuberculosis was made and the patient was started on anti-tuberculosis drugs. Considering the fact that patient's serum creatnine levels were $2 \mathrm{gm} / \mathrm{dL}$, he was put on Isoniazid (300 mg OD (once daily)), Ofloxacin (600 mg OD), Pyrazinamide (1500 mg), Ethambutol (800 mg OD), Streptomycin (750 mg OD) and was closely monitored. Patient developed hyperbilirubinaemia following which isoniazid was replaced with rifampicin (450 mg OD). Patient was later discharged with advice of follow-up after 2 months as by RNTCP (revised national tuberculosis control programme).

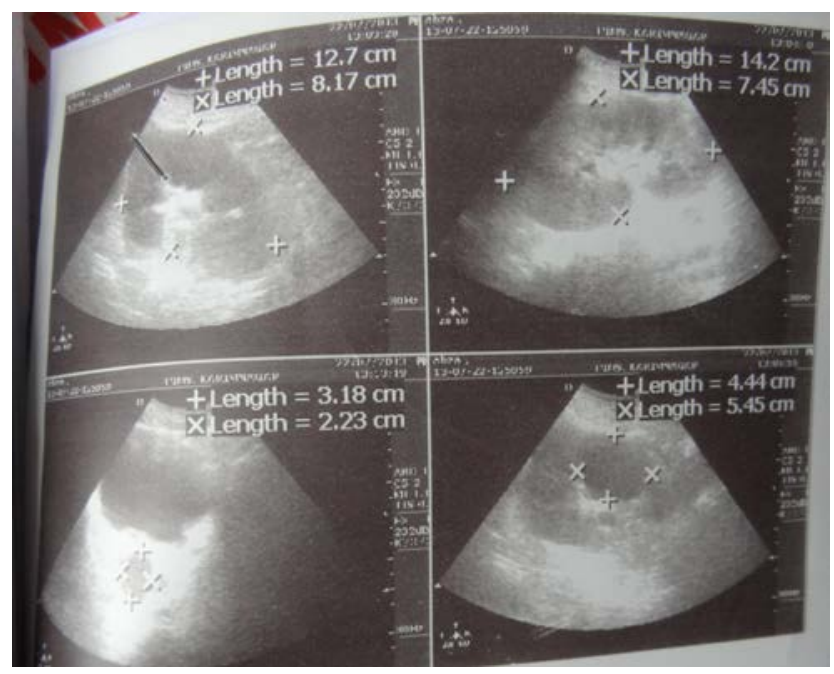

Figure 1. Ultra sound Abdomen showing kidney abnormalities

\section{Discussion}

Renal tuberculosis is a secondary tuberculosis infection which is caused due to the haematogenous dissemination during primary infection or reactivation. The cortex of the kidney is an appropriate place for lodging of bacilli since the rate of oxygenation is high. Afterwards, the infection is transmitted from the cortex to the medulla and numerous tubercles will form. At this point, cell-mediated immunity (CMI) will stop bacilli proliferation and fibrous tissue and then scar will form. But different causes such as stress, old age, immune deficiency, etc. will cause reactivation. It has been noted that there is a possibility of renal involvement during pulmonary tuberculosis and that random urine smear will be positive for AFB in few cases 
and the positivity increases on morning urine samples within 3 to 5 consecutive days [2,11]. In our patient, drug noncompliance/incomplete treatment for pulmonary tuberculosis was the predisposing factor for the severe reactivation of tuberculosis. The positive paraclincial finding in this patient was kidney abnormalities revealed on sonography that included bilateral enlarged kidney with grade 1 parenchymal changes and caliectasis and dilation of the pyelocaliceal system on both sides which are the common changes reported in renal tuberculosis $[12,13]$. Renal tuberculosis occurs concomitantly with the active pulmonary tuberculosis in $40 \%$ of cases and with silent pulmonary tuberculosis in 60\%. Sterile pyuria is the most common clinical finding and usually at this point, severe dissemination of bacilli and the resulting necrosis have occurred. Hematuria, flank pain, dysuria and sometimes involvement of other parts of the genitourinary system such as the epididymis or bladder are also reported. Severity of the bladder infection correlates with the severity of kidney infection [14,15]. A study from America has noted that though cases of pulmonary TB have been coming down there was an increase of incidences of extra-pulmonary TB since 1970's [16,17]. In this patient, respiratory symptoms and presence of pulmonary infiltration on chest x-ray confirmed the involvement of lungs with renal tuberculosis. The main paraclinical finding in our patient was the sterile pyuria associated with hematuria which was suggestive of renal tuberculosis considering the previous history of pulmonary tuberculosis of the patient.

\section{Conclusion}

Diagnosis of disseminated tuberculosis/extrapulmonary TB is not easy as it requires strong clinical and radiological suspicion, as in many cases it is difficult to demonstrate acid fast bacilli and is usually culture negative. Though many advanced and molecular methods including the polymerase chain reaction (PCR) are available, the poor and economically weak nations have been plagued by financial constraints to acquire them. Considering the fact that geographically many Asian and African countries are endemic to tuberculosis and that there is rise in the incidence of extra-pulmonary tuberculosis, MDR-TB and XDR-TB cases both in developing and developed nations, emphasis should be on early detection of disseminated tuberculosis and carefully monitored treatment to reduce the morbidity and mortality.

\section{Ethical Concerns}

Informed consent was obtained from the patient.

\section{Acknowledgements}

I sincerely acknowledge the support of the management, Prathima Institute of Medical Sciences, Karimnagar.

\section{References}

[1] Global Tuberculosis Report, 2013. http://www.who.int/tb/publications/global_report/en/ accessed on 28 January 2014.

[2] Rom WN, Garay S. Tuberculosis, W.B Saunders. USA. 2000; 47 : 609-16.

[3] Leite OHM. Tuberculosis. Probl. Gen. Surg. 2001; 18: 68-78

[4] Andre Avarese Figueiredo, Antonio Marmo Lucon, Renato Falci Junior and Miguel Srougi. Epidemiology of urogenital tuberculosis worldwide. International Journal of Urology 2008; 15 827-832.

[5] Gow JG. Genitourinary tuberculosis. In: Walsh PC, Retik AB, Vaughan ED, Wein AJ (eds). Campbell's urology. V.1, 7a edn. W. B Saunders company Philadelphia, PA, 1998; 807-836.

[6] Schubert GE, Haltaufderheide T, Golz R. Frequency of urogenital tuberculosis in an unselected autopsy series from 1928 to 1949 and 1976 to 1989. Eur. Urol. 1992; 21: 216-223.

[7] Wise GJ. Genitourinary tuberculosis: modern issues. Curr Urol Rep 2009; 10: 313-8.

[8] Smita Chandra, Harish Chandra, Neena Chauhan, Dushyant Singh Gaur, Harendra Gupta, Ved Prakash Pathak and sandeep Kumar Burathoki. Male genitourinary tuberculosis-13 years experience at a tertiary care center in India. Southeast Asian J Trop Med Public Health 2012; 43 (2): 364-369.

[9] Figueiredo AA and Lucon AM. Urogenital tuberculosis: Update and review of 8,961 cases from the world literature. Rev Urol 2008; 10: 207-217.

[10] Das P, Ahuja A, Gupta SD. Incidence, etiopathogenesis and pathological aspects of genitourinary tuberculosis in India: A journey revisited. Indian J Urol 2008; 24; 356-361.

[11] Matsumura N, Yamamoto K, Hirohashi R, Kitano S. Renal tuberculosis mimicking hydronephrosis. Intern Med 2005; 44 (7): 768.

[12] Bernaschina CP, Cabrera M, Cardona P, Colon B. Genitourinary tuberculosis: the importance of early diagnosis and management. Bol Asoc Med P R 1994; 86 (10-12): 75-80.

[13] Muttarak M, ChiangMai WN, Lojanapiwat B. Tuberculosis of the genitourinary tract: imaging features with pathological correlation. Singapore Med J 2005; 46 (10): 568-74; quiz 575.

[14] Velayati AA, Masjedi MR, Tabatabaee SJ, Zia-Zarifi A. Clinical Tuberculosis. $1^{\text {st }}$. edition. 1994; 181-219.

[15] Trasca E, Trasca ET, Buzulica R, Dragoi G, Nicolescu I. The place and the role of histological examination in diagnostic algorithm of urinary system tuberculosis. Rom J Morphol Embryol 2005; 46 (2): 105-8.

[16] Centers for Disease Control and Prevention. Reported tuberculosis in the United states, 1999, 2000, and 2001. [cited 2014 June 2] Availabe at http://WWW.cdc.gov/nchstp/tb/surv/surv.htm.

[17] Gonzalez OY, Adams G, Teeter LD, Bui TT, Musser JM, Graviss EA. Extra-pulmonary manifestations in a large metropolitan area with a low incidence of tuberculosis. Int J Tuberc Lung Dis. 2003; 7: 1178-85. 